\title{
Low-energy quenching of positronium by helium
}

\author{
Sadhan K. Adhikari, P. K. Biswas, and R. A. Sultanov \\ Instituto de Física Teórica, Universidade Estadual Paulista, 01405-900 São Paulo, São Paulo, Brazil
}

(Received 28 October 1998)

\begin{abstract}
Very-low-energy scattering of orthopositronium by helium has been investigated for the simultaneous study of elastic cross section and pickoff quenching rate using a model exchange potential. The present calculational scheme, while it agrees with the measured cross section of Skalsey et al., reproduces successfully the parameter ${ }^{1} Z_{\text {eff }}$, the effective number of electrons per atom in a singlet state relative to the positron. Together with the fact that this model potential also leads to an agreement with measured medium energy cross sections of this system, this study seems to resolve the long-standing discrepancy at low energies among different theoretical calculations and experimental measurements. [S1050-2947(99)09006-X]

PACS number(s): 34.10. $+x, 36.10$. Dr
\end{abstract}

Studies on positronium- (Ps-) impact scattering have gained momentum these days due to the availability of the ortho-Ps beam in the laboratory and its vast applicational potential coupled with the present inadequate and inconclusive understanding of its interaction dynamics with matter [1]. Ps scattering by neutral targets has posed a challenge to theoreticians on a proper accounting of experimental data as most existing theoretical works disagree with the major experimental trend.

The discrepancy figures prominently in the Ps-He system where there are many theoretical and experimental studies. The medium-energy experimental cross section shows a declining trend with decreasing energy [2] from a peak around $20 \mathrm{eV}$ for Ps-He scattering. A similar trend is also observed in Ps- $\mathrm{H}_{2}$ and Ps-Ar systems [2,3]. This trend, which is supported by the recent measurement of Skalsey et al. [4], could not be reproduced in most theoretical predictions [5-9]. At low energies, these theories and experiments $[2-4,10,11]$ on the Ps-He system are inconsistent with each other and also among themselves. For illustration, the zero-energy cross sections calculated on Ps-He by different authors vary from $3.3 \AA^{2}$ [12] to $16.54 \AA^{2}$ [13] while the measured values range from $2.3 \pm 0.4 \AA^{2}$ (at $0.915 \mathrm{eV}$ ) [4] to $11 \pm 3 \AA^{2}$ (between 0 and $0.3 \mathrm{eV}$ ) [10]. Pointing out the very reactive nature of Ps scattering and its associated convergence difficulties, a prescription for the generation of a nonlocal model exchange potential has been advocated recently and applied successfully to different electron-impact (targets $\mathrm{H}, \mathrm{He}$ ) and Ps-impact (targets: $\mathrm{H}[14,15], \mathrm{He}$ [16], $\mathrm{H}_{2}$ [17], Ar, Ne [18]) scattering problems using static exchange to three-Ps-state models. The three-Ps-state calculation for Ps-He predicts a lower zero-energy cross section of $2.42 \AA^{2}$ [16].

In this work we shed light on the above-mentioned discrepancy in the Ps-He system in conjunction with a determination of the parameter ${ }^{1} Z_{\text {eff }}$, which denotes the effective number of electrons per atom in a singlet state relative to the positron. The incident ortho-Ps $(1 s)$ atom in a triplet state with a lifetime of 142 ns can decay into three photons and is more stable than its para counterpart in a singlet state with a lifetime of $0.125 \mathrm{~ns}$ for a two-photon decay mode. However, in its interaction with matter, the positron of Ps can find an atomic electron in a spin-singlet state and the two can be annihilated by a two-photon decay mode without really forming a para-Ps atom by electron exchange. This process is termed pickoff quenching. From the experimental pickoff quenching rate the parameter ${ }^{1} Z_{\text {eff }}$ can be extracted. Theoretically ${ }^{1} Z_{\text {eff }}$ can be calculated from the wave function of the Ps-He system $\Psi\left(\mathbf{r}_{\mathbf{p}}, s_{p} ; \mathbf{r}_{\mathbf{1}}, s_{1} ; \mathbf{r}_{\mathbf{2}}, s_{2} ; \mathbf{r}_{\mathbf{3}}, s_{3}\right)$, where $\mathbf{r}$ and $s$ refer to position and spin, the suffix $p$ refers to the positron, and $i=1,2,3$ refers to the electrons. Following Barker and Bransden [7,13], the amplitude for finding the positron and one of the atomic electrons in a relative singlet state is

$$
\Phi\left(\mathbf{r}_{\mathbf{p}} ; \mathbf{r}_{\mathbf{1}} ; \mathbf{r}_{\mathbf{2}}, s_{2} ; \mathbf{r}_{3}, s_{3}\right)=\left\langle\chi_{0}\left(s_{p}, s_{1}\right) \mid \Psi\right\rangle,
$$

where $\chi_{0}$ is the singlet wave function. The parameter ${ }^{1} Z_{\text {eff }}$ is given by

$$
{ }^{1} Z_{\text {eff }}=3 \sum_{\text {spin }} \int d \mathbf{r}_{\mathbf{p}} d \mathbf{r}_{1} d \mathbf{r}_{\mathbf{2}} d \mathbf{r}_{\mathbf{3}} \delta\left(\mathbf{r}_{\mathbf{p}}-\mathbf{r}_{\mathbf{1}}\right)|\Phi|^{2}
$$

The factor 3 appears as each of the three electrons of the Ps-He system contributes equally to ${ }^{1} Z_{\text {eff }}$.

Unlike the scattering cross sections, which are determined from the asymptotic part of the Ps-He wave function, the parameter ${ }^{1} Z_{\text {eff }}$ is sensitive to the Ps-He wave function at short distances and its correct evaluation in a theoretical calculation should provide a sensitive test about its realistic nature. There is considerable discrepancy between theory and experiment in the value of the parameter ${ }^{1} Z_{\text {eff }}$. The experimental measurements have yielded ${ }^{1} Z_{\text {eff }}=0.108$ $\pm 0.01[13,19],{ }^{1} Z_{\text {eff }}=0.135 \pm 0.068[7,20]$, and ${ }^{1} Z_{\text {eff }}=0.25$ $\pm 25 \%[7,21]$, whereas different static-exchange calculations have yielded values ranging from 0.02 to $0.1[5,7,13]$. Compared to other exchange potentials, the present scheme leads to a substantially weaker repulsive exchange potential and it is expected that the present scheme will lead to a larger value of ${ }^{1} Z_{\text {eff }}$ in the Ps-He system as is demanded by experiments [19-21].

In the static-exchange approximation the Ps-He wave function is represented by the following antisymmetrized product of the internal wave functions of $\operatorname{Ps}(1 s), \phi_{\mathrm{Ps}_{\mathrm{s}}}\left(\mathbf{r}_{\mathbf{1}}\right.$ $\left.-\mathbf{r}_{\mathbf{p}}\right)$, and singlet $\mathrm{He}(1 s 1 s), \phi_{\mathrm{He}}\left(\mathbf{r}_{\mathbf{2}}, \mathbf{r}_{\mathbf{3}}\right)$, with a wave function of relative motion, $F_{\mathbf{k}}(\mathbf{R})$, and a suitable spin function:

$$
\Psi=\mathcal{A} \phi_{\mathrm{Ps}}(\mathbf{t}) \phi_{\mathrm{He}}\left(\mathbf{r}_{2}, \mathbf{r}_{3}\right) F_{\mathbf{k}}(\mathbf{R}) \chi\left(s_{1}, s_{2}, s_{3}, s_{p}\right),
$$


where $\mathbf{R}=\left(\mathbf{r}_{1}+\mathbf{r}_{\mathrm{p}}\right) / 2, \mathbf{t}=\mathbf{r}_{\mathbf{1}}-\mathbf{r}_{\mathrm{p}}, \mathcal{A}$ is the antisymmetrizer, $\mathbf{k}$ the incident Ps momentum, and $\chi$ the spin function.

On expanding $F(\mathbf{R})$ in partial waves,

$$
F_{\mathbf{k}}(\mathbf{R})=\sum_{L=0}^{\infty}(2 L+1)(k R)^{-1} F_{L}(R) P_{L}(\cos \theta),
$$

where $\theta$ is the angle between $\mathbf{k}$ and $\mathbf{R}$, the following integrodifferential equation is obtained from the Schrödinger equation:

$$
\begin{aligned}
& \left(\frac{d^{2}}{d R^{2}}+k^{2}-\frac{L(L+1)}{R^{2}}\right) F_{L}(R) \\
& \quad=\int_{0}^{\infty} V_{L}\left(R, R^{\prime}\right) F_{L}\left(R^{\prime}\right) R^{\prime 2} d R^{\prime},
\end{aligned}
$$

where $V_{L}\left(R, R^{\prime}\right)$ is the nonlocal exchange potential. The asymptotic boundary conditions for $F_{\mathbf{k}}(\mathbf{R})$ and $F_{L}(R)$ are given by

$$
\begin{aligned}
& F_{\mathbf{k}}(\mathbf{R}) \underset{R \rightarrow \infty}{\sim} \exp (i k R \cos \theta)+f(\theta) \frac{\exp (i k R)}{R}, \\
& F_{L}(R) \underset{R \rightarrow \infty}{\sim} \sin \left(k R-L \pi / 2+\delta_{L}\right),
\end{aligned}
$$

where $\delta_{L}$ is the scattering phase shift, and the scattering amplitude $f(\theta)$ is given by

$$
f(\theta)=\sum_{L=0}^{\infty}(2 L+1) \frac{\exp \left(i \delta_{L}\right) \sin \delta_{L}}{k} P_{L}(\cos \theta) .
$$

The total elastic and momentum transfer cross sections are given by

$$
\begin{gathered}
\sigma_{\mathrm{el}}\left(k^{2}\right)=\int|f(\theta)|^{2} d \Omega, \\
\sigma_{m}\left(k^{2}\right)=\int|f(\theta)|^{2}(1-\cos \theta) d \Omega,
\end{gathered}
$$

respectively. Some of the experiments provide only a lowenergy momentum-transfer cross section and hence we also calculate this observable in this study.

We employ a $\mathrm{He}(1 s 1 s)$ wave function of the following form:

$$
\begin{gathered}
\phi_{\mathrm{He}}\left(\mathbf{r}_{2}, \mathbf{r}_{3}\right)=u_{2}\left(\mathbf{r}_{2}\right) u_{3}\left(\mathbf{r}_{\mathbf{3}}\right), \\
u_{i}(\mathbf{r})=\sum_{\kappa} a_{\kappa i} \phi_{\kappa i}(\mathbf{r}),
\end{gathered}
$$

with $\phi_{\kappa i}(\mathbf{r})=\exp \left(-\alpha_{\kappa i} r\right) Y_{00}$. In the present calculation we use an accurate two-term parametrization of Eq. (12) [22]. In momentum space the model exchange potential has the following form [16]:

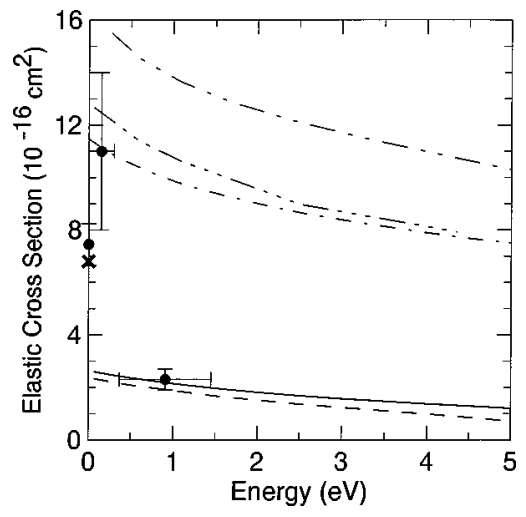

FIG. 1. Angle-integrated Ps-He cross section at low positronium energies: present momentum transfer from static-exchange model (dashed line); present elastic from static-exchange model (full line); elastic from static exchange of Ref. [7] (dashed-dotted line); of Ref. [13] (dashed-double-dotted line); of Ref. [8] (dashed-triple-dotted line); theory of Ref. [5] (cross); experiments at $0 \mathrm{eV}, 0.15 \mathrm{eV}$, and $0.9 \mathrm{eV}$ of Refs. $[11,10,4]$, respectively (solid circle).

$$
\begin{aligned}
B\left(\mathbf{k}_{\mathbf{f}}, \mathbf{k}_{\mathbf{i}}\right)= & {\left[\sum_{j} \sum_{\kappa \kappa^{\prime}} \frac{4 a_{\kappa j} a_{\kappa^{\prime} j}}{D_{\kappa \kappa^{\prime} j}} \int \phi_{\kappa^{\prime} j}^{*}(\mathbf{r})\right.} \\
& \left.\times \exp (\mathrm{i} \mathbf{Q} \cdot \mathbf{r}) \phi_{\kappa j}(\mathbf{r}) d \mathbf{r}\right] \\
& \times \int \phi_{\mathrm{Ps}}^{*}(\mathbf{t}) \exp (\mathrm{i} \mathbf{Q} \cdot \mathbf{t} / 2) \phi_{\mathrm{Ps}}(\mathbf{t}) d \mathbf{t},
\end{aligned}
$$

with $\mathbf{Q}=\mathbf{k}_{\mathbf{i}}-\mathbf{k}_{\mathbf{f}}, \quad V(\mathbf{p}, \mathbf{q})=-B(\mathbf{p}, \mathbf{q}) /\left(2 \pi^{2}\right)$, and

$$
D_{\kappa \kappa^{\prime} j}=\left[k_{f}^{2} / 4+\alpha_{\kappa j}^{2}+\beta^{2}\right],
$$

where $\phi_{\kappa j}(\mathbf{r})$ is the $\kappa$ th function of the $j$ th electron for the atomic ground state, and $\phi_{\mathrm{Ps}}(\mathbf{t})=\beta^{3 / 2} \exp (-\beta t) / \sqrt{\pi}$. The direct potential for this problem is zero, and there is a change of sign in the spin-triplet Ps-He potential below. The partialwave configuration space nonlocal potential of Eq. (5) is given by

$V_{L}\left(R, R^{\prime}\right)=\left(\frac{2}{\pi}\right)^{2} \int_{0}^{\infty} \int_{0}^{\infty} p^{2} d p q^{2} d q j_{L}(p R) V_{L}(p, q) j_{L}\left(q R^{\prime}\right)$

and

$$
V_{L}(p, q)=-\pi^{2} \int_{-1}^{1} d x P_{L}(x) V(\mathbf{p}, \mathbf{q})
$$

where $x$ is the cosine of the angle between $\mathbf{p}$ and $\mathbf{q}$. Although the model exchange potential (16) has been found to be satisfactory for calculating scattering cross sections [16], it is interesting to investigate if it is also effective in the calculation of finer scattering observables, such as the parameter ${ }^{1} Z_{\text {eff }}$. A scheme for the calculation of ${ }^{1} Z_{\text {eff }}$ for He wave functions of type (11) is given by Fraser and Kraidy [13] and we employ the same in the present calculation.

First we show our results for the low-energy elastic and momentum-transfer cross sections in Fig. 1 together with those obtained from other theories and experiments. Our trip- 


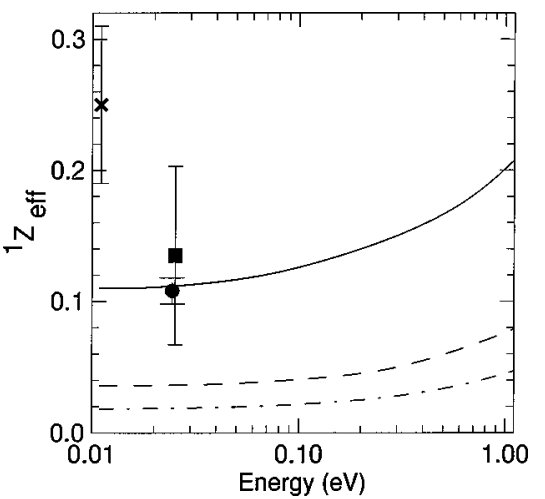

FIG. 2. The parameter ${ }^{1} Z_{\text {eff }}$ at different positronium energies: calculation including angular momenta $L=0,1,2$ of Ref. [13] (dashed-dotted line), of Ref. [7] (dashed line); calculation for $L$ $=0$ of present model (full line); the experimental points denoted by solid circle, diamond, and cross taken from Refs. [19-21], respectively.

let Ps-He scattering length is $0.87 a_{0}$, compared to $1.39 a_{0}$ obtained by Drachman and Houston [5]. The discrepancy among various results is apparent in this plot. The three experimental results for cross sections $[4,10]$ shown by solid circles vary from $2.3 \AA^{2}$ [4] through $7.45 \AA^{2}$ [11] to $11 \AA^{2}[10]$ at $0.9 \mathrm{eV}, 0 \mathrm{eV}$, and $0.15 \mathrm{eV}$, respectively. It is difficult to reconcile these three experimental results in a theoretical model. Previous static-exchange calculations $[7-9,13]$ except those of Ref. [16] all tend to support the largest cross section of Ref. [10]. The model calculation of Drachman and Houston [5] denoted by a cross in Fig. 1 is consistent with the experiment of Ref. [11]. This discrepancy has been partially resolved in the study of Ref. [16], where it has been demonstrated that the present model exchange potential is unique in being able to reproduce experimental cross sections [2,3] up to medium energies (about $60 \mathrm{eV}$ ) fairly well. Other theoretical calculations are unable to reproduce $[7,8,13]$ the experimental trend of a total cross section at different energies with a minimum around $5.1 \mathrm{eV}$. The present elastic (full line) and momentum-transfer (dashed line) static-exchange calculations are consistent with the experiment of Skalsey et al. [4].

Next we perform an $S$-wave calculation for the parameter ${ }^{1} Z_{\text {eff }}$. In Fig. 2 we plot the results for the present calculation of ${ }^{1} Z_{\text {eff }}$ at different energies. For comparison we also plot the results of a previous calculation by Barker and Bransden [7] and by Fraser and Kraidy [13] and the existing three experimental data. Although Drachman and Houston [5] did not calculate ${ }^{1} Z_{\text {eff }}$ at different energies, their low-energy value of 0.1 is in reasonable agreement with the present result of 0.11 and the experiment of Refs. $[19,20]$. The three experimental results with error bars cover the range of 0.070.31 for ${ }^{1} Z_{\text {eff }}$. Of the three experiments, the one by Duff and Heymann [19] with the smallest error bar might be the most accurate.

The much too small values of ${ }^{1} Z_{\text {eff }}$ obtained in previous calculations $[7,13]$ seem to be a consequence of a much stronger (exchange) repulsion in the elastic channel of these models. This is reflected in the zero-energy cross section or the scattering length of these calculations. For a repulsive potential the low-energy cross section increases with repul-

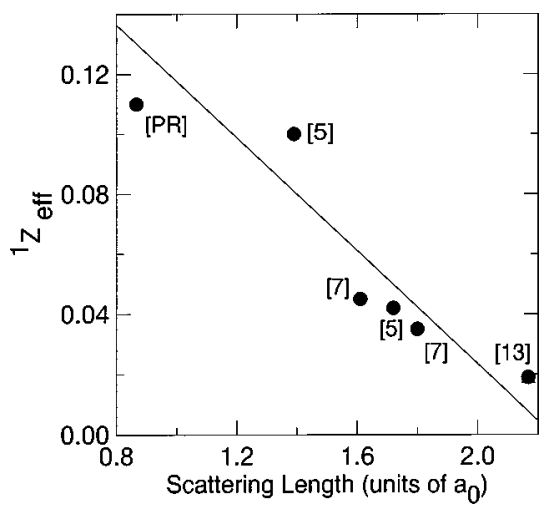

FIG. 3. The parameter ${ }^{1} Z_{\text {eff }}$ versus scattering length of various calculations denoted by a solid circle and labeled by reference numbers $[5,7,13]$. The present result is labeled by [PR]; full line denotes a linear fit.

sion, consequently the previous calculations have led to unusually large triplet scattering lengths compared to the present work. This is most clearly exhibited in a correlation exhibited in Fig. 3, where we plot ${ }^{1} Z_{\text {eff }}$ versus the triplet scattering length of different calculations. The larger the scattering length, the smaller is the ${ }^{1} Z_{\text {eff }}$. This correlation is similar to different correlations observed in the study of Ps-H scattering in Ref. [15].

We next comment on two aspects of the present calculation. First, we used a two-term helium wave function. We also repeated our calculation with the one-term helium wave function of Ref. [9] and the five-term wave function used in Ref. [16]. The results for both the cross section and ${ }^{1} Z_{\text {eff }}$ suffer insignificant change with the change of wave function. For ${ }^{1} Z_{\text {eff }}$, the different results are within the error bar of Ref. [19]; for the cross section they are also within the error bar of Ref. [4]. Hence we do not believe the present results are so peculiar as to be of no general validity. Second, we performed an $L=0$ calculation for ${ }^{1} Z_{\text {eff }}$. At experimental energies less than $0.03 \mathrm{eV}$, the effect of higher partial waves is practically zero (well within the error bar of Ref. [19]); at 1 $\mathrm{eV}$ this effect is quite small.

From the consideration above, we believe the present static-exchange calculation as well as the previous calculations [16] using the same exchange potential provide a realistic account of very-low-energy Ps-He scattering. However, the precise agreement of the static-exchange calculation with experiment is expected to be incidental. For a complete understanding of this problem, higher excited states of both Ps and $\mathrm{He}$ should be incorporated in the model. The inclusion of Ps excitation channels has been found [16] to decrease the low-energy cross sections and we might need to refit the low-energy cross sections by changing the parameters $\alpha$ and/or $\beta$ of the potential in Eq. (14), as in Refs. $[15,16]$.

In conclusion, we have used a recently suggested nonlocal model exchange potential [14-17] and applied it to the study of Ps-He scattering at low energies. We have critically examined the static-exchange calculation to see if it can account satisfactorily for [16] the measured cross sections of Refs. [2,4] and the "measured" value of the parameter ${ }^{1} Z_{\text {eff }}$ of Refs. [19,20]. The present calculation is in reasonable agreement with the calculation of Ref. [5]. However, it is difficult to reconcile the present calculation with the experiment of Ref. [21]. In a previous study [16], the present ex- 
change potential has been found to reproduce the low- to medium-energy cross sections of Refs. [2,4] well. This, coupled with the present study, seems to indicate that the ${ }^{1} Z_{\text {eff }}$ measurement of Ref. [19] and the high- and low-energy cross-section measurements of Refs. [2] and [4] are consistent with each other as well as with the present calculation which possibly provides a faithful description of low-energy Ps-He scattering. We observe a correlation between ${ }^{1} Z_{\text {eff }}$ and the triplet scattering length of various calculations (Fig. 3), which demonstrates that the smaller the scattering length, the larger is the value of ${ }^{1} Z_{\text {eff }}$. This correlation is similar to different correlations observed recently between the lowenergy Ps-H observables [15].

We thank Professor B. H. Bransden for suggesting this investigation, and for his helpful comments and encouragement. The work was supported in part by the CNPq and FAPESP of Brazil.
[1] D. W. Gidley, A. Rich, and P. W. Zitzewitz, Positron Annihilation, edited by P. G. Coleman, S. C. Sharma, and L. M. Diana (North-Holland, Amsterdam, 1982), p. 11.

[2] A. J. Garner, G. Laricchia, and A. Ozen, J. Phys. B 29, 5961 (1996).

[3] N. Zafar, G. Laricchia, M. Charlton, and A. J. Garner, Phys. Rev. Lett. 76, 1595 (1996).

[4] M. Skalsey, J. J. Engbrecht, R. K. Bithell, R. S. Vallery, and D. W. Gidley, Phys. Rev. Lett. 80, 3727 (1998).

[5] R. J. Drachman and S. K. Houston, J. Phys. B 3, 1657 (1970).

[6] S. Hara and P. A. Fraser, J. Phys. B 8, L472 (1975).

[7] M. I. Barker and B. H. Bransden, J. Phys. B 1, 1109 (1968); 2, 730 (1969).

[8] N. K. Sarkar and A. S. Ghosh, J. Phys. B 30, 4591 (1997).

[9] P. A. Fraser, Proc. Phys. Soc. London 79, 721 (1962).

[10] Y. Nagashima, T. Hyodo, K. Fujiwara, and A. Ichimura, J. Phys. B 31, 329 (1998).

[11] K. F. Canter, J. D. McNutt, and L. O. Roellig, Phys. Rev. A
12, 375 (1975).

[12] G. Peach (unpublished), as quoted in Garner et al. [2].

[13] P. A. Fraser and M. Kraidy, Proc. Phys. Soc. London 89, 533 (1966).

[14] P. K. Biswas and S. K. Adhikari, J. Phys. B 31, 3147 (1998); 31, 5403 (1998).

[15] S. K. Adhikari and P. K. Biswas, Phys. Rev. A 59, 2058 (1999).

[16] P. K. Biswas and S. K. Adhikari, Phys. Rev. A 59, 363 (1999).

[17] P. K. Biswas and S. K. Adhikari, J. Phys. B 31, L315 (1998); 31, L737 (1998).

[18] P. K. Biswas and S. K. Adhikari (unpublished).

[19] B. G. Duff and F. F. Heymann, Proc. R. Soc. London, Ser. A 270, 517 (1962).

[20] F. F. Heymann, P. E. Osmon, J. J. Veit, and W. F. Williams, Proc. Phys. Soc. London 78, 1038 (1961).

[21] L. O. Roellig and T. M. Kelly, Phys. Rev. Lett. 18, 387 (1967).

[22] F. W. Byron and C. J. Joachain, Phys. Rev. 146, 1 (1966). 